\title{
Induced Maps, Markov Extensions and Invariant Measures in One-Dimensional Dynamics
}

\section{H. Bruin}

Department of Pure Mathematics, Delft University of Technology, P.O. Box 5031, 2600 GA Delft, The Netherlands

Received: 15 September 1993 / in revised form: 21 June 1994

\begin{abstract}
A way to study ergodic and measure theoretic aspects of interval maps is by means of the Markov extension. This tool, which ties interval maps to the theory of Markov chains, was introduced by Hofbauer and Keller. More generally known are induced maps, i.e. maps that, restricted to an element of an interval partition, coincide with an iterate of the original map.

We will discuss the relation between the Markov extension and induced maps. The main idea is that an induced map of an interval map often appears as a first return map in the Markov extension. For S-unimodal maps, we derive a necessary condition for the existence of invariant probability measures which are absolutely continuous with respect to Lebesgue measure. Two corollaries are given.
\end{abstract}

\section{Statement of the Results}

Invariant probability measures are the key ingredient of Birkhoff's Ergodic Theorem, which predicts the statistical behaviour of points in a dynamical system. Especially measures that are absolutely continuous with respect to Lebesgue measure are of interest, because in that case Birkhoff's Ergodic Theorem holds for a large set in Lebesgue sense. We will abbreviate absolutely continuous invariant probability measure to acip.

Let us consider interval maps $f: I \rightarrow I$ that are piecewise continuous and piecewise monotone. A special case are the unimodal maps, i.e. continuous maps having a unique turning point $c$. In this paper we concentrate on two methods to construct an acip for $f$.

First there is the notion of induced map. Let $\left\{J_{i}\right\}_{i}$ be an interval partition of $J \subset$ $I$ such that $\left|J \backslash \bigcup_{i} J_{i}\right|=0$. ( || denotes Lebesgue measure). The map $F: \bigcup_{i} J_{i} \rightarrow J$ is an induced map of $f$, if $F$ coincides on each interval $J_{i}$ with some iterate $f^{s_{1}}$ of $f$. The integer sequence $\left\{s_{i}\right\}_{i}$ is called the stopping rule. $F$ may be constructed

The author wants to thank Sebastian van Strien for encouragement and his comments on previous versions of this paper, and also the referee for his helpful suggestions, especially for suspecting a flaw in the previous version of Lemma 2. 
in such a way that it satisfies a Markov property, i.e. $F$ preserves the partition: $F\left(J_{i}\right) \supset J_{j}$ whenever $F\left(J_{l}\right) \cap J_{j} \neq \emptyset$. If the branches of $F$ have bounded distortion and $\left|F\left(J_{l}\right)\right|$ is uniformly bounded away from $0, F$ admits an acip $m$. There is a standard way to derive from $m$ an acip $\mu$ of $f$. In order to prove that $\mu$ is indeed finite, it suffices to verify a summability condition:

$$
\sum_{i} s_{i} m\left(J_{i}\right)<\infty
$$

We like to know whether (1) is a necessary condition for the existence of an acip. To answer this question we need a second tool: the Markov extension. The Markov extension was developed by Hofbauer and Keller [H2,HK1]. (In the unimodal setting this object is also known as Hofbauer tower.) It is a way to transform the map $f: I \rightarrow I$ into a map $\check{f}: \check{I} \rightarrow \breve{I}$ that satisfies the Markov property. The price one has to pay is that $\breve{I}$ is no longer compact. This makes it hard to find an acip on $(\check{I}, \breve{f})$, but it was shown in $[\mathrm{H} 1, \mathrm{~K}]$ that if $f$ has an acip $\mu, \mu$ lifts to an acip $\breve{\mu}$ on $\check{I}$. This in itself is the wrong direction; we hoped to construct acips for $(I, f)$. But it is useful in proving that (1) must be satisfied if an acip exists. The main idea is that induced maps, under some mild conditions, correspond to first return maps in the Markov extension. Currently used induced maps, e.g. [B1,GJ,JS,M,V], fit in this framework.

First return maps are in fact the simplest kind of induced maps; they allow an easy transport of measures: An acip on $(\check{I}, \breve{f})$ guarantees an acip for a first return map, which in its turn can be projected to an acip of the induced map $(J, F)$. In this paper, we will show that condition (1) is equivalent to the existence of an acip, provided the induced map is natural in some sense. The precise definitions of natural and naturally extendible will be given at Lemma 2, Sect. 3.

Theorem 1. Let $f: I \rightarrow I$ be piecewise continuous and piecewise monotone, and let $\mu$ be an acip such that the metric entropy $h_{\mu}>0$. Assume that $f$ is conservative (see Sect. 2) with respect to $\mu$. Let $F$ be an induced map with stopping rule $\left\{s_{i}\right\}_{i}$, which preserves an acip $m$. If $F$ is natural or naturally extendible, then

$$
\sum s_{i} m\left(J_{i}\right)<\infty \text {. }
$$

Remark. We will prove Theorem 1 for unimodal maps. This is the simplest case combinatorially. Also the acip of an S-unimodal map automatically has positive metric entropy. The necessary adjustments for the general case will be given at the appropriate places as remarks.

Remark. Because we are mainly interested in acips, we have formulated Theorem 1 for acips. But in fact Theorem 1 holds for all invariant probability measures having positive metric entropy. One must be aware however, that there is a fixed relation between $\mu$ and $m$. Formula (6) shows that $\mu$ determines $m$, and vice versa.

In the next theorem, Theorem 1 is applied to a certain induced map, studied by Martens. The point $x$ is called a closest preimage of the critical point, $c$, if $f^{n}(x)=c$ for some $n$, and $f^{j}((x, c)) \not \supset c$ for $0<j<n$. The iterates $n$ for which this happens are called the cutting times. They are denoted by $S_{k}$, where we start counting at $S_{0}=1$. Denote the closest preimages to the left of $c$ by $c_{-S_{k}}$. Then the symmetric points $\hat{c}_{-S_{k}}$ of $c_{-S_{k}}$ (i.e. $f\left(c_{-S_{k}}\right)=f\left(\hat{c}_{-S_{k}}\right)$ ) are the closest preimages to the right of $c$. Let $A_{k}=\left(c_{-S_{k-1}}, c_{-S_{k}}\right)$. 
A map $f$ is called $S$-unimodal if it is $C^{3}$ unimodal and has negative Schwarzian derivative: $S f(x)=\frac{D^{3} f(x)}{D f(x)}-\frac{3}{2}\left(\frac{D^{2} f(x)}{D f(x)}\right)^{2}<0$ for all $x \neq c$. Let $l$ be the order of the critical point $c$, i.e. there exists a smooth coordinate transformation $\varphi$ such that for $x$ close to $c, f(x)=f(c)-|\varphi(x-c)|^{l} . f$ is called non-flat if $l<\infty$.

Theorem 2. Let $f$ be a non-flat $S$-unimodal map, then

$$
\sum_{k} S_{k}\left|A_{k}\right|<\infty
$$

is a necessary condition for $f$ to have an absolutely continuous invariant probability measure.

We will finish with a few corollaries. The first one gives a purely topological condition excluding acips. Let $r(n)$ be the largest integer for which $f^{r(n)}$ is monotone on $\left(c, c_{n}\right)$, where $c_{n}=f^{n}(c)$. If $r(n)$ is very large compared to $n$ (but finite), $f$ is very close to a saddle node bifurcation creating an $n$-periodic point. This almost saddle node behaviour is one of the processes that can prohibit the existence of an acip.

Corollary 1. Let $f$ be $S$-unimodal. If $\lim \sup _{n} r(n) \lambda^{n}=\infty$ for all $\lambda>0$, then $f$ admits no acip.

In [B1] we construct an explicit example of this in the long-branched setting, as we call it.

A second result involves the Fibonacci map. This is a unimodal map with a special combinatorial pattern, discussed in [LM, KN, HK2]. A Fibonacci map is characterized by the fact that the cutting times $\left\{S_{k}\right\}_{k}$ are the Fibonacci numbers: $S_{0}=1, S_{1}=2$ and $S_{k+1}=S_{k}+S_{k-1}$. Let $f_{l}$ be an S-unimodal Fibonacci map such that its critical point has order $l$. It was proved in [BKNS] that if $l$ is sufficiently large, $f_{l}$ has an absorbing Cantor set: $\omega(c)$ is a Cantor set, $f$ is not infinitely renormalizable but still $\omega(c) \supset \omega(x)$ a.e. Because also $|\omega(c)|=0[\mathrm{M}]$, an acip cannot exist. (The result in [BKNS] holds for $C^{2}$ maps actually.) So the next corollary follows from [BKNS] and [M].

Corollary 2. An S-unimodal Fibonacci map admits no acip if $l$ is sufficiently large.

For simplicity of the proof however, we want to present it as a corollary of Theorem 2. Also there may be values for $l$ that are large enough for Corollary 2 to hold, but not large enough for $f_{l}$ to have an absorbing Cantor set. We conjecture that there exist $l_{0}<l_{1}$ such that:

- For $l<l_{0}, f_{l}$ has an acip. (An acip is proved to exist if $l<2+\varepsilon, \varepsilon>0$ sufficiently small $[\mathrm{KN}]$.)

- For $l_{0}<l<l_{1}, f_{l}$ has no acip, and no absorbing Cantor set either.

- For $l>l_{1}, f_{l}$ has an absorbing Cantor set.

\section{Invariant Measures and Induced Maps}

Let $X$ be an interval or a countable union of intervals. If $f: X \rightarrow X$ is given, then $F: Y \subset X \rightarrow Y$, is called an Markov map if

i) there is a countable interval partition $\left\{Y_{i}\right\}_{i}$ of $Y$ such that $\left|Y \backslash \bigcup_{j} Y_{j}\right|=0$.

ii) If $F\left(Y_{j}\right) \cap Y_{i} \neq \emptyset$ then $F\left(Y_{j}\right) \supset Y_{i}$. In other words $f$ preserves the partition. $F$ is an induced Markov map if additionally 
iii) there exist integers $s_{i}$ such that $F_{\mid Y_{l}}=f_{\mid Y_{l}}^{s_{1}}$.

iv) $F_{\mid Y_{l}}$ is monotone for each $i$.

According to this definition, the first return map of $f$ to $Y$ need not be an induced Markov map. Conditions ii) and iv) need not be satisfied. If $f$ is not conservative, condition i) may fail as well: We call $f$ conservative with respect to $\mu$ if for every forward invariant set $A$, either $\mu(A)$ or $\mu\left(A^{c}\right)=0$. (Notice that this definition is somewhat different from the usual definition in ergodic theory, which states that the wandering set has zero measure.) Without the conservativity constraint, $F$ need not be defined a.e. Still many first return maps are induced Markov maps.

Lemma 1. Let $f: X \rightarrow X$ be conservative with respect to an acip $\mu$. Let $Y \subset X$, $\mu(Y)>0$ and let $F: Y \subset X \rightarrow Y$ be the first return map. Then $(Y, F)$ is conservative with respect to $\mu$ and $\frac{\mu}{\mu(Y)}$ is an acip on $(Y, F)$.

Proof. Suppose by contradiction $(Y, F)$ is not conservative. Then there is a forward $F$-invariant set $B \subset Y$ which is half measured in $Y$, i.e. $\mu(B), \mu(Y \backslash B)>0$. Let $A=$ $\bigcup_{l \in \mathbb{N}} f^{i}(B) . A$ is forward $f$-invariant and half measured, because $A \cap(Y \backslash B)=\emptyset$. This contradicts conservativity of $(X, f)$.

Let $Y_{k}=\left\{x \in Y \mid F(x)=f^{k}(x)\right\}$. By conservativity $Y=\bigcup_{k} Y_{k}$ modulo null sets. Let $B \subset Y$. For any $k, f^{l}\left(Y_{k}\right) \cap B=\emptyset$ if $0<i<k$. Moreover, $\mu\left(B \backslash \bigcup_{k} F\left(Y_{k}\right)\right)=$ 0 . Let $B_{0}=B$ and $B_{j}=f^{-1}\left(B_{j-1}\right) \backslash Y$, so $B_{J}=\left\{x \in X \mid f^{j}(x) \in B, f^{i}(x) \notin Y\right.$ for $0 \leqq i<j\}$. By $f$-invariance of $\mu, \mu(B)=\sum_{j \geqq 0} \mu\left(f^{-1}\left(B_{j}\right) \cap Y\right)=\sum_{j \geqq 1} \mu\left(F^{-1}(B)\right.$ $\left.\cap Y_{j}\right)=\mu\left(F^{-1}(B)\right) \cdot \frac{\mu}{\mu(Y)}$ is clearly a probability measure.

If a Markov map satisfies certain metric properties, it has an acip. This result is often called a Folklore Theorem. We will use a version based on distortion. If $f$ is a $C^{1}$ map on an interval $J$, then the distortion $\operatorname{dist}(f, J)=\sup _{x, y \in J} \log \left|\frac{D f(x)}{D f(y)}\right|$.

Theorem 3 (Folklore Theorem). If a Markov map $F: \bigcup_{j} Y_{j} \rightarrow Y$, has the additional properties:

- $F^{n}$ has bounded distortion for all $n \geqq 1$ and on each interval of monotonicity,

- $F\left(Y_{j}\right)=Y$ for all $j$,

then $F$ has an acip $m$ and $d m$ is bounded and bounded away from zero.

For the proof of Theorem 3 we refer to [MS]. We emphasize that similar results can be obtained under weaker conditions. In many cases, e.g. the induced map that emerges from the next theorem, boundedness of distortion follows from the Koebe Principle [MMS] and extendibility of the branches. A branch of $f^{n}$ is the restriction of $f^{n}$ to an interval $J$ on which $f^{n}$ is monotone. If $\delta>0$ is given, a branch $f_{\mid J}^{n}$ is extendible with respect to $\delta$ if there exists $T \supset J$ such that $f_{\mid T}^{n}$ is monotone and both components of $f^{n}(T \backslash J)$ have length $\geqq \delta\left|f^{n}(J)\right|$.

In the next theorem, we use a measure theoretic interpretation of attractor. Let $A \subset I$. The basin $B(A)$ of $A$ is the set $\{x \mid \omega(x) \subset A\} . A$ is an attractor if $|B(A)|>0$, and there is no proper subset of $A$ with the same property. In the S-unimodal case the possible attractors were classified in [BL1]: An attractor can be i) a periodic attractor, ii) a periodic interval, iii) a solenoidal attractor (e.g. the Feigenbaum attractor), or iv) an absorbing Cantor set. 
Theorem 4 (Martens). Suppose $f$ is non-flat, S-unimodal and has no periodic attractor, solenoid attractor or absorbing Cantor set. Then there exist symmetric neighbourhoods $\mathscr{V} \supset \mathscr{U} \ni c$ with the following properties:

$-f^{k}(\partial \mathscr{U}) \subset \partial \mathscr{V}$ for some $k$ and $f^{j}(\mathscr{U}) \cap \mathscr{V}=\emptyset$ for $0<j<k$.

$-f^{j}(\partial \mathscr{V}) \notin \mathscr{V}$ for all $j \geqq 0$.

- for Lebesgue a.e. $x \in I$, there exist neighbourhoods $T_{x} \supset J_{x} \ni x$ and $s(x) \in \mathbb{N}$ such that $f^{s(x)}\left(J_{x}\right)=\mathscr{U}, f^{s(x)}\left(T_{x}\right)=\mathscr{V}$ and $f_{\mid T_{\mid}}^{s(x)}$ is monotone.

In particular, it can be proved that $f$ is conservative if and only if $f$ has no attractor of type i), iii) or iv). Moreover, these kind of attractors have zero Lebesgue measure (see $[\mathrm{GJ}]$ and $[\mathrm{M}]$ ). The proof of Theorem 4 can be found in [M, MS].

The first two properties of Theorem 4 imply that $f^{\prime}(\partial \mathscr{U}) \notin \mathscr{U}$. For this reason $\partial \mathscr{U}$ is called nice. The sets $\left\{J_{x} \mid x \in \mathscr{U}\right\}$ form a countable partition of $\mathscr{U}$. Let us write this partition as $\left\{U_{l}\right\}_{i}$. Due to the niceness of $\partial \mathscr{U}$, these intervals are indeed pairwise disjoint. The map $F: \bigcup_{i} U_{i} \rightarrow \mathscr{U}$ defined as $F(x)=f^{s(x)}(x)$ for the appropriate iterate $s(x)$ is an induced Markov map. All branches of $F$ are extendible to branches that cover $\mathscr{V}$. In order to apply the Folklore Theorem, the branches must have bounded distortion, but this is guaranteed by the Koebe Principle. Because the Schwarzian derivative is negative, the Koebe Principle may be used.

Suppose $F: \bigcup_{i} J_{j} \rightarrow J,\left|J \backslash \bigcup_{i} J_{i}\right|=0$, is an induced Markov map having the stopping rule $\left\{s_{i}\right\}_{i}$ and an acip $m$. Then there is a standard way to find an acip $\mu$ for $f$ :

$$
\mu(A)=\sum_{l} \sum_{j=0}^{s_{i}-1} m\left(J_{i} \cap f^{-J}(A)\right) .
$$

The proof that $\mu$ is absolutely continuous and invariant is straightforward. Applied to the whole interval $I$, (3) yields $\mu(I)=\sum_{l} s_{l} m\left(J_{l}\right)$. So if

$$
\sum_{l} s_{l} m\left(J_{i}\right)<\infty,
$$

then $\mu$ is finite. If $m$ has a bounded density, then one can replace (1) by

$$
\sum_{l} s_{i}\left|J_{i}\right|<\infty \text {. }
$$

In the next section we will show that (1) is often a necessary condition for the existence of a finite measure too.

\section{The Markov Extension of $f$}

Let $f: I \rightarrow I$ be unimodal. Following Hofbauer and Keller [HK1] we discuss the Markov extension $(\breve{I}, \breve{f}) . \breve{I} \subset I \times \mathbb{N}$ consists of a countable union of intervals $\breve{D}_{n}=$ $\left(D_{n}, n\right)$. The end-points of $D_{n}$ are forward iterates of $c$, one of which is $c_{n} . D_{1}=$ $\left[c, c_{1}\right]$ and inductively

$$
D_{n+1}= \begin{cases}f\left(D_{n}\right) & \text { if } D_{n} \not \ngtr c, \\ {\left[c_{1}, c_{n+1}\right]} & \text { if } D_{n} \ni c .\end{cases}
$$

By definition of closest preimages, the second case only happens if $n=S_{k}$ for some $k$. So if $S_{k-1}<n \leqq S_{k}, D_{n}=\left[c_{n}, c_{n-S_{k-1}}\right]$ and $D_{n} \subset D_{n-S_{k-1}}$. In particular $c \in$ 
$D_{S_{k}} \subset D_{S_{k}-S_{k-1}}=D_{S_{k^{\prime}}}$, for some cutting time $S_{k^{\prime}}<S_{k}$. Therefore one can define the kneading map $Q: \mathbb{N} \rightarrow \mathbb{N} \cup \infty$ as $S_{Q(k)}=S_{k}-S_{k-1}$. $\breve{f}$ acts on $\check{I}$ as follows: If $(x, n)=\check{x} \in \check{D}_{n}$ then

$$
\check{f}(\check{x})= \begin{cases}(f(x), n+1) & \text { if } x \text { and } c_{n} \text { lie on the same side of } c, \\ \left(f(x), S_{Q(k)}+1\right) & \text { if } x \text { and } c_{n} \text { lie on different sides of } c,\end{cases}
$$

where in the latter case $n=S_{k}$. Let $\pi: \breve{I} \rightarrow I$ be the natural projection, then $f \circ \pi=$ $\pi \circ \check{f}$. Observe that if $\breve{J} \subset \check{I}$ is an interval,

$$
\check{f}_{\mid \check{J}}^{k} \text { is continuous, whenever } f_{\mid \pi(\check{J})}^{k} \text { is monotone. }
$$

Hence $\check{f}^{k}$ can move $\check{J}$ upwards or downwards in the Markov extension, but $\check{J}$ is never cut into pieces if $f^{k} \mid \pi(\breve{J})$ is monotone. For more details see [B2, H2, HK1].

Remark. We briefly sketch the construction of the Markov extension for the case that $f$ is piecewise continuous and piecewise monotone. A generalization of kneading maps is possible, but needlessly complicated for the scope of this paper. Let $Z_{l}, i=1, \ldots, N$, be the maximal intervals on which $f$ is continuous and monotone. $\breve{I}$ is the disjoint union $\bigsqcup_{M \in \mathscr{M}} M$, where $\mathscr{M}$ is a collection of intervals defined as follows: $f\left(Z_{i}\right) \in \mathscr{M}$ for $i=1, \ldots, N$, and if $M \in \mathscr{M}$, then also $f\left(M \cap Z_{i}\right) \in \mathscr{M}$. The action $\check{f}$ on $\check{I}$ is defined as: if $\check{x} \in M \cap Z_{i}$, then $\breve{f}(\check{x})=f(x) \in$ $f\left(M \cap Z_{l}\right)$. Observe that $\breve{f}: \check{I} \rightarrow \check{I}$ is indeed a Markov map, and that (5) is still satisfied.

Theorem 5. Let $f$ be an S-unimodal map, having an acip $\mu$. Then there exists an invariant probability measure $\breve{\mu}$ on $(\breve{I}, \breve{f})$ such that $\mu=\breve{\mu} \circ \pi^{-1}$. Moreover, $\check{f}$ is conservative.

Proof. The proof of the first statement can be found in [H1], under the assumption that the metric entropy $h_{\mu}>0$. However, we prefer to sketch the argument from $[\mathrm{K}]$. In that paper Keller generalizes results from [H1].

Let inc: $\left[c_{2}, c_{1}\right] \rightarrow\left(\left[c_{2}, c_{1}\right], 2\right)$ be the natural inclusion, take $\mu=\check{\mu}_{1} \circ$ inc and

$$
\check{\mu}_{n}=\frac{1}{n} \sum_{i=0}^{n-1} \check{\mu}_{1} \circ \check{f}^{-i}
$$

The measure $\mu$ on $I$ lifts to a measure on $\check{I}$, i.e. the sequence $\left\{\check{\mu}_{n}\right\}_{n}$ has a weak accumulation point $\check{\mu}_{\infty}$ which is an acip. The invariance of the weak accumulation point is clear. Notice that by invariance of $\mu, \breve{\mu}_{n}\left(\pi^{-1}(A)\right)=\mu(A)$ for each $n$. This yields the absolute continuity of $\breve{\mu}$ if $\mu$ is absolutely continuous.

The assumption $h_{\mu}>0$ is used to show that 0 is not a weak accumulation point of $\left\{\breve{\mu}_{n}\right\}_{n}$. But if $f$ is S-unimodal and $h_{\mu}=0$, then $f$ is essentially injective on the support of $\mu$. One can show that $c$ is a density point of this support [BL2]. So $h_{\mu}$ is automatically positive. Birkhoff's Ergodic Theorem implies that if $f$ has an acip, $f$ and $\breve{f}$ are both conservative with respect to $\mu$ and $\check{\mu}$.

Remark. If $f$ is piecewise continuous, piecewise monotone, Theorem 5 is still valid. In $[\mathrm{K}]$ no restrictions of this kind are made. However, we have to state the assumption $h_{\mu}>0$ explicitly. 
We want to tie the notions of Markov extension and induced map together. Let us consider the following diagram,

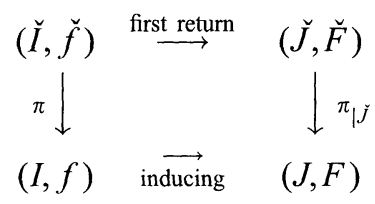

and ask ourselves to what extent it is well-defined. The projection $\pi_{\mid \breve{J}}$ and the first return map $\breve{F}: \breve{J} \rightarrow \breve{J}$ are well-defined as soon as $\breve{J}$ is defined. We will try to choose $\check{J}$ such that it is compatible with the induced map $F: J \rightarrow J$. By this we mean that $\pi_{\mid \breve{J}} \circ \breve{F}=F \circ \pi_{\mid \breve{J}}$. In other words, $\breve{J} \subset \pi^{-1}(J)$ is such that the return time of $\check{x} \in \pi^{-1}(x) \cap \check{J}$ is independent of the level $\check{D}_{k}$. If we succeed in doing this, then it is easy to transport measures. The acip $\mu$ on $(I, f)$ lifts to $\breve{\mu}$ on $(\check{I}, \check{f})$ by Theorem 5. Due to Lemma $1, \check{m}=\frac{\check{\mu}}{\check{\mu}(\check{J})}$ is an acip on $(\breve{J}, \breve{F})$, and because (6) commutes, $m=\check{m} \circ \pi_{\mid \breve{J}}^{-1}$ is an acip on $(J, F)$. Note also that $(J, F)$ is conservative.

Presently we formulate conditions on the induced Markov map that will guarantee that (6) commutes. Let $F$ be an induced Markov map $F: \bigcup_{l} J_{l} \rightarrow J$ with stopping rule $s_{l} . F$ is called natural if

i) for every $i \in \mathbb{N}$ and $0<k<s_{i}$, there exists no set $A$ such that both $A \cap J_{i} \neq \emptyset$ and $f^{k}$ maps $A$ monotonically onto $f^{s_{1}}\left(J_{j}\right)$ for any $j \in \mathbb{N}$.

Often one is interested in induced maps with extendible branches. For the induced map from Theorem 4, or for instance the induced map obtained in [JŚ], this allows the use of the Koebe Principle. Let $T_{l} \supset J_{i}$ be the domains of the extensions: $f_{\mid T_{l}}^{s_{i}}$ is monotone. $F$ is naturally extendible if,

ii) all branches of $F$ are extendible,

iii) $f^{s_{l}}\left(T_{i}\right) \supset T_{j}$ whenever $f^{s_{i}}\left(J_{i}\right) \supset J_{j}$, and

iv) for every $i \in \mathbb{N}$ and $0<k<s_{l}$, there exists no set $A$ such that $A \cap J_{i} \neq \emptyset$, $f^{k}\left(J_{i}\right) \cap f^{s^{\prime}}\left(J_{j}\right) \neq \emptyset$ and $f^{k}$ maps $A$ monotonically onto $f^{s_{j}}\left(T_{j}\right)$ for any $j \in \mathbb{N}$.

Briefly stated, $F$ is natural (naturally extendible) if the stopping rule is as small as possible, and the branch-domains are as large as possible.

Lemma 2. If the induced Markov map is natural or naturally extendible, then there exists $\breve{J}$ such that (6) commutes.

Proof. Define $\check{F}_{\mid \pi^{-1}\left(J_{l}\right)}=\check{f}_{\mid \pi^{-1}\left(J_{l}\right)}$ and set $\breve{J}=\bigcup_{k \geqq 1} \check{F}^{k}(\operatorname{inc}(J))$. We need to show that $\check{F}$ is the first return map to $\check{J}$. Let $\check{J}_{l}=\pi^{-1}\left(J_{l}\right) \cap \check{D}_{a}$ be such that $\check{J}_{i} \subset \check{J}$. Formula (5) implies that $\pi\left(\check{D}_{a}\right) \supset J_{l}$, and if $F$ is naturally extendible, property iii) even gives $\pi\left(\breve{D}_{a}\right) \supset T_{i}$. Again by (5), and the definition of $\breve{J}, \check{f}_{\mid \breve{J}_{l}}^{s_{l}}$ is a continuous and, if $F$ is naturally extendible, extendible return to $\breve{J}$.

Let us show that it is also a first return. Suppose that there exists $0<k<s_{l}$ such that $\check{f}^{k}\left(\check{J}_{i}\right) \cap \breve{J} \neq \emptyset$. Because $k<s_{i}, \check{f}_{\mid \breve{J}_{l}}^{k}$ is continuous, so $\check{f}^{k}\left(\check{J}_{i}\right) \subset \check{D}_{b}$, for some $b \in \mathbb{N}$. Suppose by contradiction that $\check{f}^{k}\left(\check{J}_{l}\right) \cap \pi^{-1}\left(f^{S_{I}}\left(J_{j}\right)\right) \neq \emptyset$ for some $j$. By definition of $\check{J}, \pi\left(\check{D}_{b}\right) \supset f^{s_{1}}\left(J_{J}\right)$. Because $(\check{I}, \check{f})$ satisfies the Markov property, 
there exists $\check{B}$ such that $\check{J}_{i} \subset \check{B} \subset \check{D}_{a}$ and $\check{f}^{k}(\check{B})=\check{D}_{b}$. In particular, there exists $\breve{A} \subset \breve{B}$ such that $\breve{J}_{i} \cap \breve{A} \neq \emptyset$ and $\check{f}^{k}(\breve{A})=\pi^{-1}\left(f^{s}\left(J_{j}\right)\right) \cap \check{D}_{b}$. Hence $F$ cannot be natural, because $\pi(\breve{A}) \cap J_{l} \neq \emptyset$ and $f^{k}$ maps $\pi(\check{A})$ monotonically onto $f^{s_{I}}\left(J_{j}\right)$. If $F$ is naturally extendible, we change $J_{j}$ into $T_{j}$, and use the same argument.

Remark. In this proof we only used the Markov property of $(\check{I}, \check{f})$ and $(5)$. So Lemma 2 also holds for piecewise continuous, piecewise monotone maps.

Theorem 6. If $\mu$ is an acip on $(I, f)$ such that $f$ is conservative and (6) commutes, then $\sum_{i} s_{i} m\left(J_{i}\right)<\infty$ and

$$
\frac{1}{\sum s_{i} m\left(J_{i}\right)}=\check{\mu}(\check{J})=\lim _{n \rightarrow \infty} \frac{1}{n} \#\left\{0 \leqq i<n \mid \check{f}^{l}(\check{x}) \in \check{J}\right\} \check{\mu} \text {-a.e. }
$$

Proof of Theorem 6. We have seen that $\check{\mu}$ and $m$ are acips on $(\check{I}, \check{f})$ and $(J, F)$ respectively and that $\check{f}$ and $F$ are conservative. $|J|>0$ and by conservativity also $\breve{\mu}(\breve{J})>0$. Indeed, if $\breve{\mu}(\breve{J})=0$, then $\bigcup_{j \geqq 0} \check{f}^{-j}(\breve{J})$ is backward invariant and zero $\check{\mu}$-measured. On the other hand, as $|\breve{J}|>0, \bigcup_{j \geqq 0} \check{f}^{-j}(\breve{J})=\check{I}$ modulo null sets. This is a contradiction. So it suffices to prove $(7)$, assuming that $\check{\mu}(\breve{J})>0$.

Birkhoff's Ergodic Theorem immediately yields the second equality of (7). Write $s(x)=s_{i}$ if $x \in J_{i}$. Let $V_{k}=\{x \in J \mid s(x)=k\}$ and let $\left\{n_{j}(x)\right\}_{j}$ be such that $F^{j}(x)=$ $f^{n_{j}(x)}(x)$ for all $j \geqq 0$. $\left\{n_{j}(x)\right\}_{j}$ is defined $\mu$-a.e. As (6) commutes, $\breve{V}_{k}=\pi_{\mid \breve{J}}^{-1}\left(V_{k}\right)=$ $\left\{\check{x} \in \breve{J} \mid \check{F}(\check{x})=\check{f}^{k}(\check{x})\right\}$. If $\left\{\check{n}_{j}(\check{x})\right\}$, for $\check{x} \in \check{J}$ is such that $\check{F}^{j}(\check{x})=\check{f}^{n_{j}(\check{x})}(\check{x})$, then simply $\check{n}_{j}(\check{x})=n_{j}\left(\pi_{\mid \check{J}}(\check{x})\right) \check{\mu}$-a.e.

Because $\check{F}$ is the first return map to $\check{J}, \#\left\{0 \leqq i<\check{n}_{j}(\check{x}) \mid \check{f}^{i}(\check{x}) \in \check{J}\right\}=j$. Hence for $\check{\mu}$-a.e. $\check{x} \in \pi_{\mid \check{J}}^{-1}(x)$,

$$
\begin{aligned}
\sum_{i} s_{i} m\left(J_{i}\right) & =\int_{J} s(y) d m(y)=\lim _{j \rightarrow \infty} \frac{1}{j} \sum_{i=0}^{j-1} s\left(F^{l}(x)\right) \\
& =\lim _{j \rightarrow \infty} \frac{n_{j}(x)}{j}=\lim _{j \rightarrow \infty} \frac{\check{n}_{j}(\check{x})}{j} \\
& =\frac{1}{\lim _{j \rightarrow \infty} \frac{1}{\check{n}_{j}(\check{x})} \#\left\{0 \leqq i<\check{n}_{j}(\check{x}) \mid \check{f}^{i}(\check{x}) \in \check{J}\right\}} \\
& =\frac{1}{\check{\mu}(\check{J})} .
\end{aligned}
$$

It follows that $\mu$ exists if and only if $\sum_{l} s_{i} m\left(J_{l}\right)<\infty$.

Proof of Theorem 1. Combine Theorem 6 and Lemma 2.

Proof of Theorem 2. Let $F$ be the induced map that emerges from Theorem 4. Due to the conditions on $\partial \mathscr{U}$ and $\partial \mathscr{V}, F$ is naturally extendible. The Folklore Theorem guarantees that $F$ has an acip $m$ with density $0<m_{0}<d m<m_{1}<$ $\infty$. Let $\mathscr{U}=\bigcup_{i} U_{l}$ modulo null sets. Then if $U_{i} \subset\left(c_{-} S_{k}, \hat{c}_{-} S_{k}\right), s_{i}>S_{k}$, simply because $f^{j}\left(U_{i}\right) \not \supset \mathscr{U}$ for $j \leqq S_{k}$. Hence $\sum_{i} s_{i} m\left(U_{i}\right)>m_{0} \sum_{l} s_{i}\left|U_{i}\right|>m_{0} \sum S_{k}\left|A_{k}\right|$. Now use Theorem 1. 


\section{Corollaries}

In this last section we prove the corollaries stated in Sect. 1. Corollary 1 relies on the notion of almost saddle nodes. At a saddle node, the central branch of $f^{n}$ is tangent to the diagonal. The point of contact $q$ is an $n$-periodic one-sided attractor. Its immediate basin is $[q, \hat{q}]$, but on the other side of $q$ still an infinite sequence of closest preimages, accumulating on $q$, exists. If the central branch of $f^{n}$ is almost tangent to the diagonal, the closest preimages retain an intermittent behaviour, which causes $\left|c-c_{-S_{k}}\right|$ to be large compared to $S_{k}$. This is the key idea in the proof of Corollary 1.

Proof of Corollary 1. We know a priori that there exists $\lambda>0$ such that, unless $f$ has a periodic attractor, $\left|c-c_{n}\right|>\lambda^{n}$ for all $n \geqq 1$. Indeed, if this were not the case, then for every $C>0$, we can find $n$ such that $\left|c-c_{n}\right|^{l-1} C l 2^{l} L^{n-1} \leqq 1$. Here $L=\max _{x \in I}|D f(x)|$ and $l$ is the order of the critical point. Let $x$ be such that $c_{n} \in(c, x)$ and $|c-x|=2\left|c-c_{n}\right|$, then

$$
\begin{aligned}
\left|f^{n}(c)-f^{n}(x)\right| & \leqq\left|\int_{c}^{x} D f^{n}(z) d z\right| \\
& \leqq|c-x| c l|c-x|^{l-1} L^{n-1} \\
& \leqq\left|c-c_{n}\right| C l 2^{l}\left|c-c_{n}\right|^{l-1} L^{n-1} \\
& \leqq\left|c-c_{n}\right| .
\end{aligned}
$$

Hence $f^{n}((c, x)) \subset(c, x)$, yielding a periodic attractor. (Notice that in fact $\lambda \geqq$ $L^{-1 /(l-1)}$. So if we have a bound for $L$, we can weaken the assumption accordingly.)

$r(n)=S_{k}$ if and only if $c_{n} \in A_{k} \cup \hat{A}_{k}$. Choose $M>0$ arbitrary, take $n$ such that $r(n) \lambda^{n}>M$ and let $S_{k^{\prime}}=r(n)$ correspondingly. Since $c_{n} \in A_{k^{\prime}} \cup \hat{A}_{k^{\prime}}$, $\left|c-c_{-S_{h^{\prime}-1}}\right| \geqq \lambda^{n}$, so

$$
\sum_{k} S_{k}\left|A_{k}\right| \geqq \sum_{k \geqq k^{\prime}} S_{k^{\prime}}\left|A_{k}\right| \geqq S_{k^{\prime}}\left|c-c_{-S_{k^{\prime}}}\right| \geqq r(n) \lambda^{n} \geqq M .
$$

As $M$ was arbitrary, this concludes the proof.

Proof of Corollary 2. Let $f_{l}$ be an S-unimodal Fibonacci map such that the order of the critical point is $l$. The cutting times grow asymptotically as $\gamma^{k}$, where $\gamma$ is the golden ratio $\frac{\sqrt{5}-1}{2}$. We may assume that Theorem 4 applies. Otherwise there is an attractor of zero Lebesgue measure, and therefore no acip whatsoever. For $k$ large, $\frac{\left|c-c_{-}\right|}{\left|c-c-s_{h-1}\right|}$ is bounded away from 0 if $l>2$ and this quotient tends to 1 as $l \rightarrow \infty$. The details of these estimates can be found in [BKNS, KN]. Choose $l$ so large that for $k$ sufficiently large $\frac{\left|c-c_{-}\right|}{\left|c-s_{k}\right|} \geqq \beta>\frac{1}{\gamma}$. It follows that $\left|c-c_{-S_{k-1}}\right| \geqq C \beta^{k}$ for all $k$ and some $C>0$. Checking the summability condition of Theorem 2,

$$
\sum_{k} S_{k}\left|A_{k}\right| \geqq S_{k^{\prime}} \sum_{k \geqq k^{\prime}}\left|A_{k}\right|=S_{k^{\prime}}\left|c_{-S_{k^{\prime}-1}}-c\right| \geqq C(\gamma \beta)^{k^{\prime}},
$$

for any $k^{\prime}$. Now take the limit $k^{\prime} \rightarrow \infty$. 


\section{References}

[B1] Bruin, H.: Topological conditions for the existence of invariant measures for unimodal maps. Erg. Th. and Dyn. Sys. 14, 433-451 (1994)

[B2] Bruin, H.: Invariant measures of interval map. Thesis, Delft, 1994

[BKNS] Bruin, H., Keller, G., Nowicki, T., van Strien, S.: Absorbing Cantor sets in dynamical systems: Fibonacci maps. Preprint Stonybrook (1994/2)

[BL1] Blokh, A.M., Lyubich, M.Yu.: Measurable dynamics of S-unimodal maps of the interval. Ann. Scient. Éc. Norm. Sup. 24, 545-573 (1991)

[BL2] Blokh, A.M., Lyubich, M.Yu.: Attractors of the interval. Banach Center Publ. 23, $427-$ 442 (1986)

[GJ] Guckenheimer, J., Johnson, S.: Distortion of S-unimodal maps. Ann. of Math. 132, 71$130(1990)$

[H1] Hofbauer, F.: On intrinsic ergodicity of piecewise monotonic transformations with positive entropy. Israel J. of Math. 34, 213-237 (1979)

[H2] Hofbauer, F.: The topological entropy of a transformation $x \mapsto a x(1-x)$. Monath. Math. 90, 117-141 (1980)

[HK1] Hofbauer, F., Keller, G.: Quadratic maps without asymptotic measure. Commun. Math. Phys. 127, 319-337 (1990)

[HK2] Hofbauer, F., Keller, G.: Some remarks on recent results about S-unimodal maps. Ann. Inst. Henri Poincaré, Physique théorique 53, 413-425 (1990)

[JŚ] Jakobson, M., Światek, G.: Metric properties of non-renormalizable S-unimodal maps, I. Induced expansion and invariant measures. Erg. Th. and Dyn. Sys. 14, 721-755 (1994)

[K] Keller, G.: Lifting measures to Markov extension. Monath Math. 108, 183-200 (1989)

[KN] Keller, G., Nowicki, T.: Fibonacci maps re(al)-visited. Preprint Univ. of Erlangen. (1992)

[LM] Lyubich, M., Milnor, J.: The Fibonacci unimodal map. Jour. AMS 6, $425-457$ (1993)

[M] Martens, M.: Interval Dynamics. Thesis, Delft, 1990

[MMS] Martens, M., de Melo, W., van Strien, S.: Julia-Fatou-Sullivan theory for real onedimensional dynamics. Acta Math. 168, 273-318 (1992)

[MS] de Melo, W., van Strien, S.: One-dimensional dynamics. Ergebnisse der Mathematic und ihrer Grenzgebiete, Berlin, Heidelberg, New York: Springer, 1993

[V] Vargas, E.: Markov partitions in non-hyperbolic interval dynamics. Commun. Math. Phys. 138, 521-535 (1991)

Communicated by J.-P. Eckmann 